\title{
Bioaccumulation and Toxicity of Carbon Nanoparticles Suspension Injection in Intravenously Exposed Mice
}

\author{
Ping Xie ${ }^{1}$, Sheng-Tao Yang ${ }^{2, *}$ (D), Tiantian $\mathrm{He}^{3}$, Shengnan Yang ${ }^{2}$ and Xiao-Hai Tang ${ }^{3, *}$ \\ 1 State Key Laboratory of Oral Diseases, West China College of Stomatology, Sichuan University, \\ Chengdu 610041, China; xieping318@aliyun.com \\ 2 College of Chemistry \& Environment Protection Engineering, Southwest Minzu University, \\ Chengdu 610041, China; 18641460881@163.com \\ 3 Chongqing Lummy Pharmaceutical Co., Ltd., Chongqing 401123, China; tthe2017@126.com \\ * Correspondence: yangst@pku.edu.cn (S.-T.Y.); pharmmateceo@aliyun.com (X.-H.T.); \\ Tel.: +86-28-8552-2269 (S.-T.Y.); +86-28-8550-3334 (X.-H.T.)
}

Received: 9 November 2017; Accepted: 23 November 2017; Published: 29 November 2017

\begin{abstract}
Carbon nanoparticles suspension injection (CNSI) has been widely used in tumor drainage lymph node mapping, and its new applications in drug delivery, photothermal therapy, and so on have been extensively investigated. To develop new clinical applications, the toxicity of CNSI after intravenous exposure should be thoroughly investigated to ensure its safe use. Herein, we studied the bioaccumulation of CNSI in reticuloendothelial system (RES) organs and the corresponding toxicity to mice. After the intravenous injection of CNSI, no abnormal behavior of mice was observed during the 28-day observation period. The body weight increases were similar among the exposed groups and the control group. The parameters of hematology and serum biochemistry remained nearly unchanged, with very few of them showing significant changes. The low toxicity of CNSI was also reflected by the unchanged histopathological characteristics of these organs. The injection of CNSI did not induce higher apoptosis levels either. The slight oxidative stress was observed in RES organs at high dosages at day 7 post-exposure. The implication to the clinical applications and toxicological evaluations of carbon nanomaterials is discussed.
\end{abstract}

Keywords: carbon nanoparticles suspension injection; biodistribution; biosafety; Raman spectroscopy; nanotoxicity

\section{Introduction}

Carbon nanomaterials, such as carbon nanoparticles, carbon quantum dots, fullerene, carbon nanotubes (CNTs), and graphene, have attracted great research interest in the past decades $[1,2]$. The unique structures and fantastic properties make carbon nanomaterials suitable for biomedical applications [3]. In particular, carbon nanomaterials have been found to have great potential in theranostics, including bioimaging, diagnosis, drug delivery, gene therapy, photothermal therapy, and so on [4-6]. For example, carbon quantum dots could be used for sentinel lymph node imaging and photodynamic therapy $[7,8]$. Graphene-based drug delivery systems have been used in cancer treatment [9]. The laboratory results show the bright future of carbon nanomaterials in clinical applications. Although more efforts are required to commercialize them, carbon nanomaterials are expected to benefit public health in the future.

Among these novel carbon nanomaterials, carbon nanoparticles suspension injection (CNSI) is the only one that has been produced on a large scale and applied in clinical treatments. Annually, over 100,000 patients receive CNSI injections during oncological surgery, and the numbers keep increasing 
quickly [10]. Currently, CNSI is only used for the lymphatic mapping and for distinguishing the parathyroid gland [11-14]. First, CNSI migrates fast in lymphatic vessel and is trapped in lymph nodes to stain them black. Researchers have used diverse cancer models to establish the high performance of CNSI in tumor drainage lymph node (TDLN) mapping. For instance, Li et al. dissected the lymph nodes after staining with CNSI in advanced gastric cancer [11]. Wu et al. achieved the TDLN mapping of early breast cancer by using CNSI [12]. Zhu et al. performed central lymph node dissection for patients with papillary thyroid carcinoma using CNSI as the tracer [13]. Second, CNSI does not stain the parathyroid gland during thyroid carcinoma surgery, thus reducing the risk of false resection. Gu et al. used CNSI to identify parathyroid from thyroid and lymph nodes during surgery, which largely preserved the parathyroid glands from false resection [14].

It should be noted that CNSI shares many characteristics with other carbon nanomaterials, so CNSI might be used in more biomedical areas beyond the mapping of lymph nodes. In fact, CNSI adsorbs drugs well and could be used as a drug carrier. For example, Xie et al. found that CNSI adsorbed epirubicin and doxorubicin efficiently [15]. Yang et al. reported that CNSI significantly enhanced the drug concentration in lymph nodes and reduced the plasma drug concentration in the regional lymphatic chemotherapy of epirubicin [16]. Other potential applications of CNSI might include photothermal therapy, gene delivery, and use as an immune adjuvant. However, before new explorations and applications are proposed, the toxicity of CNSI is an urgent issue that needs to be addressed $[17,18]$. Because the previous applications of CNSI only focused on regional injection, the toxicity of CNSI has not been evaluated upon exposure through other pathways. For clinical applications, the most important exposure is intravenous injection. Unfortunately, the biosafety information of intravenously exposed CNSI is not available to date.

Although the toxicity of CNSI is unknown, the literature results of other carbon nanomaterials indicate that the toxicity of carbon nanomaterials is generally low after intravenous injection and the properties of carbon nanomaterials regulate their biosafety $[19,20]$. Previously, we reported that CNTs accumulated in body during the 90-day observation period and only induced slight toxicity and oxidative stress [21]. Another similar formulation to CNSI was carbon quantum dots (polyethylene glycol (PEG) functionalized carbon nanoparticles), which were nontoxic to mice after intravenous exposure [22]. In other reports, some carbon nanomaterials were found to be toxic. For examples, graphene oxide (GO) was found to induce macrophage nodule formation in the lungs after intravenous injection at $2.1 \mathrm{mg} / \mathrm{kg}$ bodyweight [23]. After dextran functionalization, the tolerable dose increased to $125 \mathrm{mg} / \mathrm{kg}$, and toxicity was observed only at $250 \mathrm{mg} / \mathrm{kg}$ or higher [24]. Separately, Zhang and co-workers found that CNTs induced hepatotoxicity to mice after intravenous injection in a 2-month investigation [25]. Therefore, it is very likely that CNSI has low toxicity, but this hypothesis requires more evidence.

In this study, we systematically investigated the biodistribution and toxicity of CNSI in mice after intravenous injection. The bioaccumulation of CNSI was studied by using Raman spectroscopy and optical microscopy. The behaviors were recorded and the body weights were measured. The hematology and serum biochemistry were analyzed to reveal potential function changes. The histopathological changes were investigated under an optical microscope. The apoptosis of tissues was assayed by the terminal deoxynucleotidyl transferase-mediated dUTP nick-end labeling (TUNEL) method. The oxidative stress was also measured to reveal the possible toxicological mechanism. The implications to the biosafety evaluations and the new applications of CNSI are discussed.

\section{Results and Discussion}

\subsection{Characterization of CNSI}

According to the preparation procedure of CNSI, carbon black (CH40, Mitsubishi Chemical Co., Tokyo, Japan) containing carbon nanoparticles ( $21 \mathrm{~nm}$ in diameter) was washed by ethyl acetate and nitric acid before dispersion in polyvinyl pyrrolidone (PVP). CNSI was a dark black dispersion of 
carbon nanoparticles that remained stable for months of storage at room temperature. As indicated in Figure 1a, CNSI was composed of small carbon particles, revealed under transmission electron microscopy (TEM, Autoflex, Bruker, Bonn, Germany). The diameters of these particles were in the range of $10-50 \mathrm{~nm}$, which further aggregated into larger aggregates. According to the dynamic light scattering (DLS) analyses, CNSI had a hydrodynamic radius of $189 \mathrm{~nm}$, reflecting the aggregation of carbon nanoparticles. The chemical components and functional groups of carbon nanoparticles without adding suspending reagent PVP were characterized by X-ray photoelectron spectroscopy (XPS) and infrared spectroscopy (IR). There were 93.8 at \% of carbon atoms in the carbon core of CNSI. Other elements included 5.0 at $\% \mathrm{O}$ and 1.2 at $\% \mathrm{~N}$. According to the $\mathrm{C} 1 \mathrm{~s}$ XPS spectrum, $54.6 \%$ of the carbon atoms were $\mathrm{sp}^{2}$ carbon. The other carbon atoms were $30.9 \% \mathrm{of} \mathrm{sp}^{3}$ carbon and $14.5 \%$ of $\mathrm{C}-\mathrm{O}$ bond or shake-up signal [17]. For the broad band at $289.0 \mathrm{eV}$, it was assigned to $\mathrm{C}-\mathrm{O}$ and other shake-up components, resulting in an extremely broad full width at half maximum (FWHM). It should be noted that XPS only detected the signals of the surficial atoms (several nanometers in depth). Other methods such as energy dispersive spectroscopy and elementary analysis are also recommended for future studies. The IR analysis indicated the presence of $-\mathrm{OH}\left(3440 \mathrm{~cm}^{-1}\right)$, aromatic $\mathrm{C}-\mathrm{C}\left(1640 \mathrm{~cm}^{-1}\right)$, and $1090 \mathrm{~cm}^{-1}(\mathrm{C}-\mathrm{O})$. No signal was observed at around $1730 \mathrm{~cm}^{-1}$, indicating the lack of $\mathrm{C}=\mathrm{O}$. Overall, the characterization data suggested the CNSI sample contained no toxic impurity and was properly oxidized for dispersing, thus suitable for the following distribution and toxicity assays.

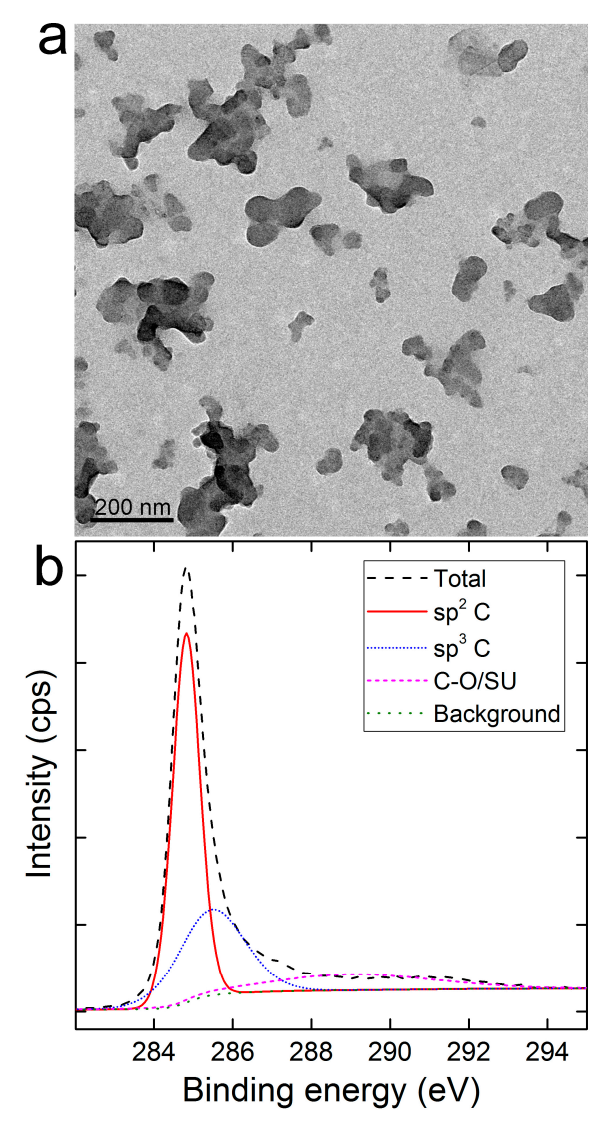

Figure 1. Transmission electron microscopy (TEM) image (a) and C1s X-ray photoelectron spectroscopy (XPS) spectrum (b) of carbon nanoparticles suspension injection (CNSI).

\subsection{Bioaccumulation of CNSI in Reticuloendothelial System (RES)}

According to the literature, in many cases, carbon nanomaterials were trapped in the liver, spleen, and lungs after intravenous injection $[20,26]$. Small particles of carbon nanomaterials might also 
accumulate in kidneys for renal excretion [22,27]. Here, we checked the hematoxylin-eosin (HE) staining slides of the heart, liver, spleen, kidneys, lungs, and axillary lymph node. As shown in Figure 2, many large dark brown particles were observed in the liver (marked by white arrows). These large aggregates were too big for cellular uptake and most likely existed in the intercellular space. The smaller ones might enter the Kupffer cells [26]. The particles were smaller in the spleen, but still easy to recognize. For the lungs, only a very faint brown color was distinguished after careful checking. The brown colored spots were assigned to CNSI aggregates, which were not found in the control group. In the other three organs, no brown colored spots were found, suggesting the absence of CNSI. To confirm the accumulation of CNSI in mice, the grounded tissue samples were analyzed by Raman spectroscopy, in which the $G$ band at around $1590 \mathrm{~cm}^{-1}$ was a typical signal of $\mathrm{sp}^{2}$ carbon structure. It should be noted that CNSI had a highly disordered structure, so the $\mathrm{G}$ band was much weaker than other typical $\mathrm{sp}^{2}$ carbon nanomaterials, such as CNTs and graphene. The $785 \mathrm{~nm}$ laser was used to avoid the strong autofluorescence of tissues. Clearly, G-band signals were found in the liver, spleen, and lungs. Consistent with the hemoglobin (HE) staining, stronger signals were found in the liver and spleen, while a very weak signal was observed for the lungs (slightly higher than the background signal). Nevertheless, the results of optical microscopy and Raman spectroscopy collectively indicated the accumulation of CNSI in RES organs. According to the literature, carbon nanomaterials are easily bound with proteins in the bloodstream [19]. Thus, CNSI might be recognized by opsonin after entering the blood circulation, where opsonization led to the capture in RES organs [28]. Most of the CNSI particles would likely be trapped by phagocytic cells, e.g. Kupffer cells [26], and the extremely large aggregates were stopped in the intercellular space. The very low pulmonary accumulation indicated that CNSI was well dispersed in the blood circulation to escape the filtration by the pulmonary capillary [26], which was consistent with the observations that CNSI dispersed well in water, saline, serum, and cell culture medium. The RES accumulations also reminded us to investigate the potential toxicity of CNSI to the accumulating organs. In addition, no CNSI was detected in other tissues or excreta by Raman spectroscopy due to the lower sensitivity comparing to other quantitative methods, such as isotope labeling and fluorescence imaging [26].

\subsection{Toxicity Evaluations}

During the 28-day observation period, no mouse died and no obvious abnormal behavior was observed. The body weight increases were similar $(p>0.05)$ among the control group and the CNSI-exposed groups (Table 1). The normal behaviors and body weight increases suggested that CNSI had low toxicity to mice after intravenous injection. This was consistent with the clinical observations that only several cases in all the treated patients (over 500,000) showed very short hyperpyrexia after the regional injection of CNSI [10]. The low toxicity of CNSI was then verified by the assays of hematology, serum biochemistry, histopathology, and apoptosis.

First, the hematological parameters were analyzed after exposure to CNSI. The data are listed in Table 2. At 1 day post-exposure, most parameters remained normal after the intravenous injection of CNSI. Significant changes were only observed for hemoglobin $(\mathrm{HB})$ and mean corpuscular volume (MCV). The HB increased from $145 \mathrm{~g} / \mathrm{L}$ (control group) to $155 \mathrm{~g} / \mathrm{L}$ ( $80 \mu \mathrm{g}$ group) and $156 \mathrm{~g} / \mathrm{L} \mathrm{(320} \mathrm{\mu \textrm {g }}$ group). The MCV increased from $49 \mathrm{fL}$ (control group) to $56 \mathrm{fL}$ (80 $\mu \mathrm{g}$ group). Even when the changes were statistically significant, the increases were generally small. Therefore, the hematological toxicity of CNSI was negligible at 1 day post-exposure. The situations were similar for the data at 7 days and 28 days, and only one or two datum points showed statistical changes. At 7 days, the mean corpuscular hemoglobin (MCHC) and MCV values of the $320 \mu \mathrm{g}$ group were slightly larger than those of the control group. At 28 days, only the mean platelet volume (MPV) of the $160 \mu \mathrm{g}$ group was higher than that of the control group. No apparent dose-dependent or time-dependent trends were observed for these changes. Thus, the hematology indicated that CNSI was nearly nontoxic to mice after intravenous injection. 

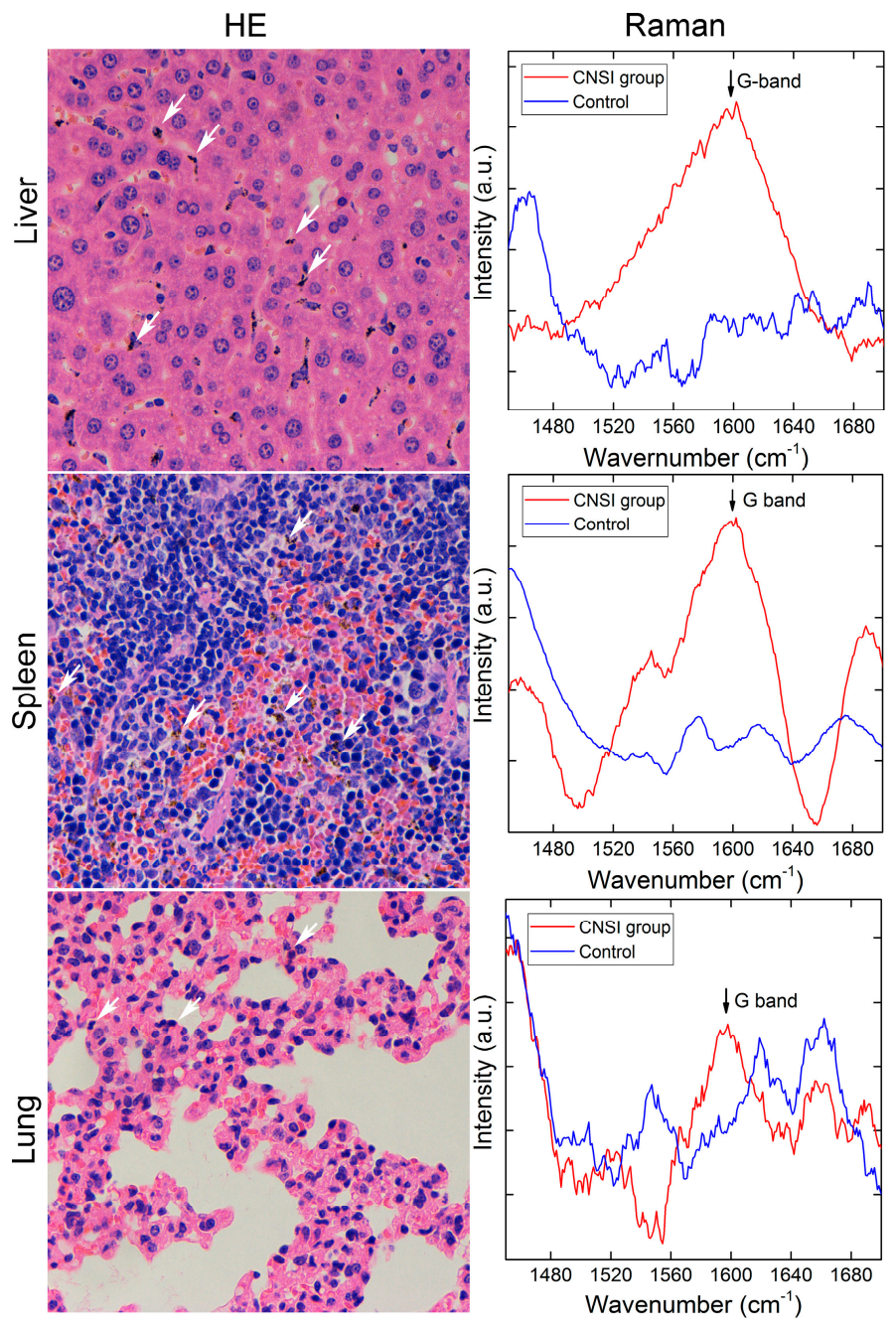

Figure 2. Bioaccumulations of CNSI in reticuloendothelial system (RES) organs, including the liver, spleen, and lungs. The dark aggregates of CNSI are indicated by white arrows $(400 \times)$.

Table 1. Bodyweight increases of the mice exposed to CNSI post intravenous exposure. Data represent means $\pm \operatorname{SD}(n=6)$.

\begin{tabular}{ccccc}
\hline & Control (g) & $\mathbf{8 0} \boldsymbol{\mu g}$ Group (g) & 160 $\boldsymbol{\mu g}$ Group (g) & 320 $\boldsymbol{\mu g}$ Group (g) \\
\hline Day 1 & $26.5 \pm 1.3$ & $25.5 \pm 0.7$ & $26.5 \pm 1.5$ & $26.3 \pm 1.3$ \\
Day 7 & $28.7 \pm 2.5$ & $28.5 \pm 1.9$ & $29.3 \pm 2.8$ & $28.3 \pm 2.4$ \\
Day 28 & $32.5 \pm 3.1$ & $33.4 \pm 3.7$ & $34.2 \pm 4.9$ & $33.5 \pm 3.4$ \\
\hline
\end{tabular}

The serum biochemistry also confirmed the nontoxic nature of CNSI after intravenous exposure (Figure 3). Most parameters remained unchanged during the 28-day period. Total bilirubin (TBIL) showed decreases at 1 day, which recovered at 7 days and increased at 28 days. No significant increase was observed for lactate dehydrogenase (LDH) at 1 day post-exposure. A decrease of LDH level was found at 7 days for the $320 \mu \mathrm{g}$ group. Alanine aminotransferase (ALT) and aspartate aminotransferase (AST) are very sensitive indicators for hepatic toxicity, yet only AST showed a meaningful increase at 7 days for the $80 \mu \mathrm{g}$ group. This indicated the low toxicity of CNSI to the liver. Aspartate aminotransferase (ALP) had slight increases at 1 day and 7 days, which were eliminated at 28 days. Urea (Ur) only had an increase at 28 days for the $160 \mu \mathrm{g}$ group. Creatinine $(\mathrm{Cr})$ had no change among all groups. Again, the lack of serve changes of serum biochemistry parameters and the absence of a dose-/time-dependent effect suggested the low toxicity of CNSI after intravenous injection. 
Table 2. Hematological parameters of the mice exposed to CNSI post intravenous exposure. Data represent means $\pm \operatorname{SD}(n=6)$.

\begin{tabular}{|c|c|c|c|c|c|c|c|c|c|c|c|c|}
\hline & \multicolumn{4}{|c|}{$1 \mathrm{~d}$} & \multicolumn{4}{|c|}{$7 \mathrm{~d}$} & \multicolumn{4}{|c|}{$28 \mathrm{~d}$} \\
\hline & Control & $80 \mu \mathrm{g}$ & $160 \mu \mathrm{g}$ & $320 \mu \mathrm{g}$ & Control & $80 \mu \mathrm{g}$ & $160 \mu \mathrm{g}$ & $320 \mu \mathrm{g}$ & Control & $80 \mu \mathrm{g}$ & $160 \mu \mathrm{g}$ & $320 \mu \mathrm{g}$ \\
\hline PLT $\left(10^{9} / \mathrm{L}\right)$ & $474 \pm 63$ & $486 \pm 81$ & $478 \pm 126$ & $459 \pm 98$ & $422 \pm 116$ & $578 \pm 135$ & $457 \pm 138$ & $421 \pm 69$ & $444 \pm 108$ & $332 \pm 113$ & $377 \pm 63$ & $382 \pm 52$ \\
\hline $\mathrm{MCHC}(\mathrm{g} / \mathrm{L})$ & $353 \pm 52$ & $321 \pm 23$ & $364 \pm 38$ & $339 \pm 33$ & $360 \pm 50$ & $326 \pm 24$ & $316 \pm 30$ & $313 \pm 3^{*}$ & $297 \pm 15$ & $304 \pm 33$ & $313 \pm 22$ & $305 \pm 23$ \\
\hline $\mathrm{HB}(\mathrm{g} / \mathrm{L})$ & $145 \pm 7$ & $155 \pm 6^{*}$ & $150 \pm 7$ & $156 \pm 9 *$ & $153 \pm 7$ & $154 \pm 6$ & $152 \pm 4$ & $149 \pm 12$ & $148 \pm 17$ & $142 \pm 37$ & $149 \pm 4$ & $152 \pm 6$ \\
\hline $\operatorname{MCV}(\mathrm{fL})$ & $49 \pm 5$ & $56 \pm 3 *$ & $49 \pm 5$ & $52 \pm 5$ & $49 \pm 6$ & $52 \pm 4$ & $56 \pm 4$ & $57 \pm 1 *$ & $56 \pm 5$ & $58 \pm 8$ & $53 \pm 5$ & $56 \pm 6$ \\
\hline $\operatorname{WBC}\left(10^{9} / \mathrm{L}\right)$ & $4.4 \pm 1.9$ & $5.0 \pm 0.8$ & $6.3 \pm 2.5$ & $4.5 \pm 1.0$ & $8.0 \pm 3.0$ & $5.4 \pm 3.4$ & $5.1 \pm 1.9$ & $5.0 \pm 2$ & $7.6 \pm 2.8$ & $6.1 \pm 2.7$ & $6.0 \pm 2$ & $6.9 \pm 2.7$ \\
\hline $\operatorname{RBC}\left(10^{12} / \mathrm{L}\right)$ & $8.5 \pm 0.6$ & $8.6 \pm 0.4$ & $8.5 \pm 0.4$ & $9.0 \pm 0.2$ & $8.8 \pm 0.6$ & $9.1 \pm 0.3$ & $8.7 \pm 0.3$ & $8.4 \pm 0.7$ & $9.0 \pm 0.9$ & $8.2 \pm 1.9$ & $9.0 \pm 0.4$ & $8.9 \pm 0.6$ \\
\hline $\mathrm{MCH}(\mathrm{pg})$ & $17.2 \pm 0.9$ & $18.0 \pm 0.7$ & $17.7 \pm 0.3$ & $16.9 \pm 0.6$ & $17.4 \pm 0.6$ & $17.0 \pm 0.6$ & $17.5 \pm 0.4$ & $17.8 \pm 0.4$ & $16.5 \pm 1.0$ & $17.3 \pm 0.7$ & $16.6 \pm 0.4$ & $17.2 \pm 1.1$ \\
\hline RDW (\%) & $18.1 \pm 1.4$ & $17.1 \pm 1.8$ & $17.9 \pm 2.4$ & $18.4 \pm 2.2$ & $17.5 \pm 1.4$ & $19.5 \pm 1.3$ & $16.4 \pm 1.1$ & $15.7 \pm 1.6$ & $16.6 \pm 2.9$ & $14.0 \pm 1.2$ & $16.0 \pm 1.8$ & $15.3 \pm 1.6$ \\
\hline MPV (fL) & $7.7 \pm 0.3$ & $7.8 \pm 0.3$ & $8.0 \pm 0.5$ & $7.5 \pm 0.2$ & $7.6 \pm 0.4$ & $7.3 \pm 0.2$ & $7.4 \pm 0.3$ & $7.4 \pm 0.2$ & $7.4 \pm 0.1$ & $7.7 \pm 0.4$ & $7.8 \pm 0.4$ * & $7.6 \pm 0.3$ \\
\hline PDW (\%) & $9.1 \pm 0.6$ & $9.0 \pm 0.5$ & $9.5 \pm 0.8$ & $8.7 \pm 0.5$ & $8.8 \pm 0.8$ & $8.3 \pm 0.3$ & $8.4 \pm 0.5$ & $8.4 \pm 0.5$ & $8.3 \pm 0.2$ & $8.8 \pm 0.6$ & $9.0 \pm 0.8$ & $8.7 \pm 0.7$ \\
\hline
\end{tabular}

${ }^{*} p<0.05$ compared with control group. PLT, platelet; MCHC, mean corpuscular hemoglobin concentration; HB, hemoglobin; MCV, mean corpuscular volume; WBC, white blood cell count; RBC, red blood cell count; MCH, mean corpuscular hemoglobin; RDW, red cell distribution width; MPV, mean platelet volume; PDW, platelet distribution width 


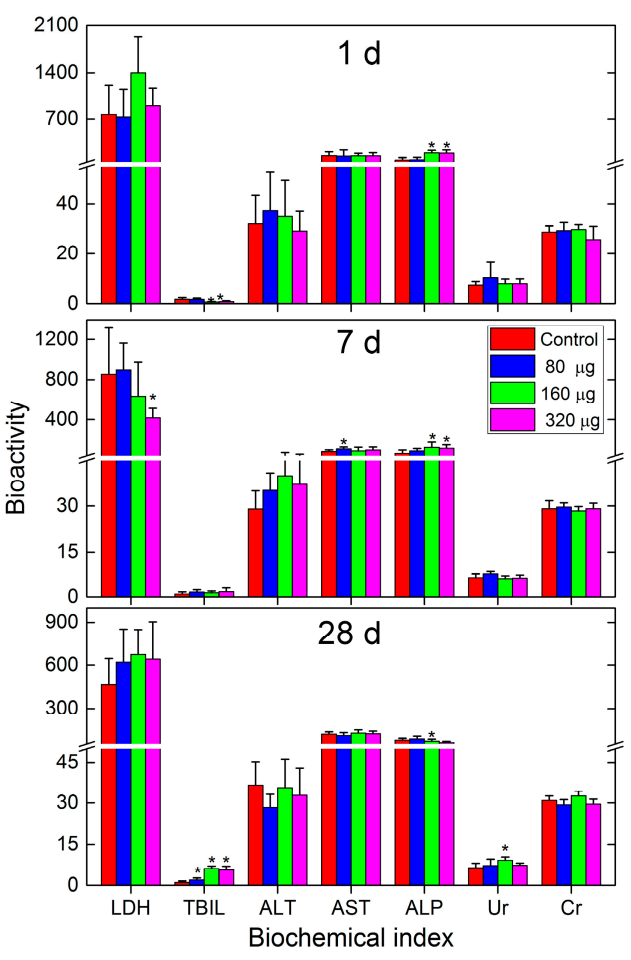

Figure 3. Serum biochemical parameters of the mice exposed to CNSI post intravenous exposure. Data represent means $\pm \operatorname{SD}(n=6) .{ }^{*} p<0.05$ compared with the control group.

The lack of functional changes after CNSI exposure was consistent with the histopathological observations (Figure 4), suggesting that CNSI did not induce organic damage after intravenous injection. No obvious histopathological change was observed for the liver, spleen, or lungs upon the HE staining under optical microscope at 1 day [29], 7 days [29], and 28 days. No steatosis, necrosis, or hydropic degeneration were presented in the exposed hepatic sections. Typical splenic unit and lymphocyte were presented in the spleen sections for the control group and the CNSI-exposed groups. No inflammatory cell infiltration occurred in the lung sections, which was widely observed for CNTs and graphene $[21,30,31]$. This was due to the high dispersibility of CNSI and small particle sizes.

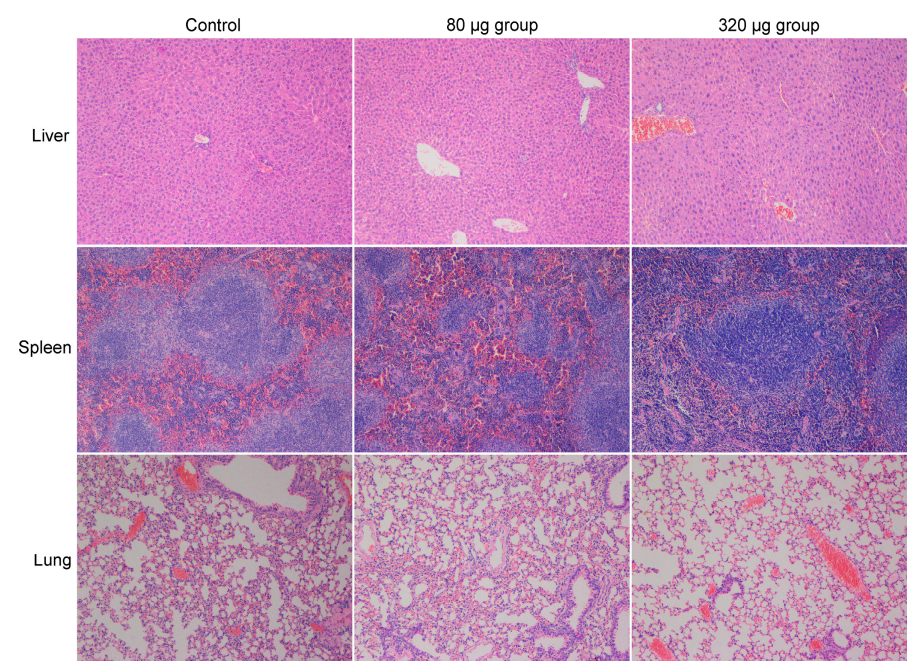

Figure 4. Histopathological observations of the mice exposed to CNSI at 28 days post intravenous exposure $(100 \times)$. 
Apoptosis is a widely observed toxic symptom in the toxicity studies of carbon nanomaterials [32]. Using the TUNEL method, we checked the apoptosis levels of mice after the intravenous exposure to CNSI at 1 day [33], 7 days [33], and 28 days (Figure 5). The brown stained nuclei were counted for comparison. In the liver sections, the hepatic cells had blue cell nuclei, suggesting the absence of apoptosis. The Kupffer cells seemed to have higher apoptosis levels, but the situations were similar among the control and the CNSI-exposed groups. For the spleen, the splenic unit was stained blue, while the lymphocyte showed slight apoptosis. Again, no meaningful difference was found among the control and CNSI-exposed groups. The nuclei in the lung sections were partially stained brown, but the apoptosis levels were similar among the three groups. Overall, CNSI did not induce apoptosis in the RES organs after intravenous exposure. This was consistent with the literature results that carbon nanomaterials induced apoptosis in vitro rather than in vivo after intravenous injection [21].

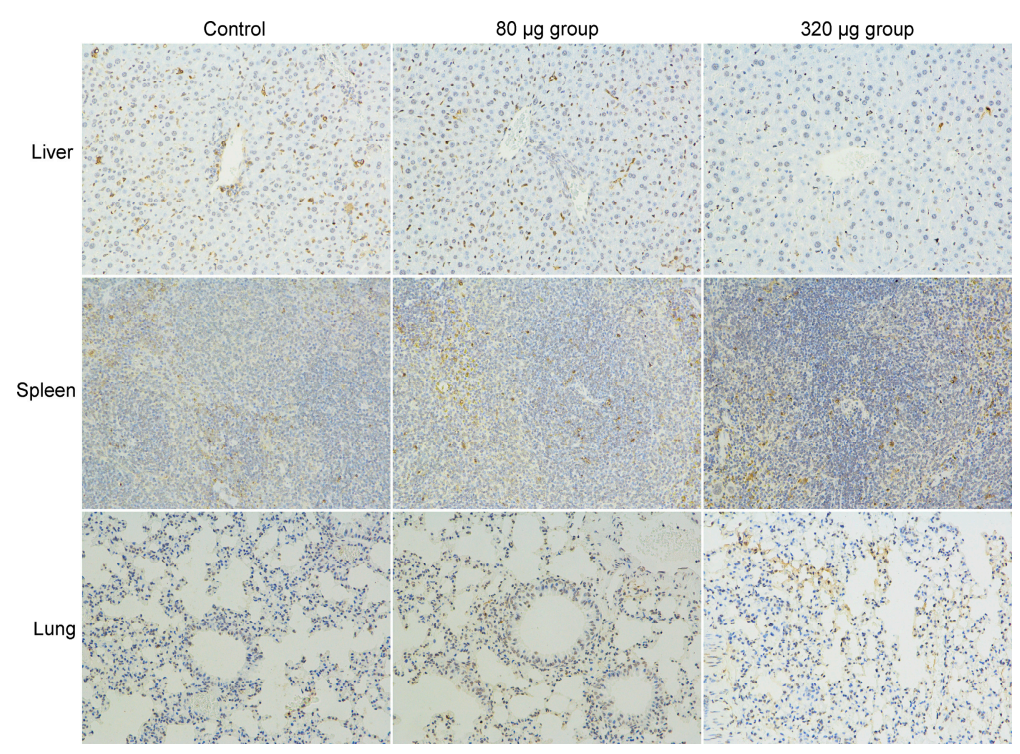

Figure 5. Apoptosis analyses of the mice exposed to CNSI at 28 days post intravenous exposure by the terminal deoxynucleotidyl transferase-mediated dUTP nick-end labeling (TUNEL) method $(200 \times)$.

Oxidative stress is usually regarded as the toxicological mechanism of carbon nanomaterials [34]. Oxidative stress is more sensitive than other toxicological indicators to reflect the potential hazards of carbon nanomaterials. Here, we measured the superoxide dismutase (SOD), catalase (CAT), and malondialdehyde (MDA) levels to investigate the potential oxidative stress caused by CNSI in mice (Figure 6). At 1 day post-exposure, apart from the fact that the MDA decreased in the lung of the $160 \mu \mathrm{g}$ group, no change was observed, suggesting the absence of oxidative stress in the short term. However, oxidative stress occurred at 7 days post-exposure. The liver had higher SOD and CAT levels at 7 days in the $320 \mu \mathrm{g}$ group, but the MDA levels remained unchanged. The spleen and lungs had increased levels of all three indicators, suggesting more oxidative stress in the spleen and lungs than in liver. The oxidative stress seemed to be alleviated at 28 days, where the levels of indicators were lower than those of the control group in the spleen and lungs. Only liver samples showed higher MDA levels in the $160 \mu \mathrm{g}$ group and the $320 \mu \mathrm{g}$ group. In a word, these results suggested that CNSI could incite oxidative stress in RES organs at 7 days, but the oxidative stress was alleviated at 28 days. The oxidative stress of CNSI was similar to that of other carbon nanomaterials. For example, CNTs induced oxidative stress in the liver and lung after intravenous injection [21]. Other carbon nanomaterials, such as GO and graphene quantum dots, did not induce oxidative stress after intravenous exposure $[23,35]$. Together with our results, the available data indicated that the oxidative stress depended on the properties of carbon nanomaterials and its rules of regulation require further investigations. 


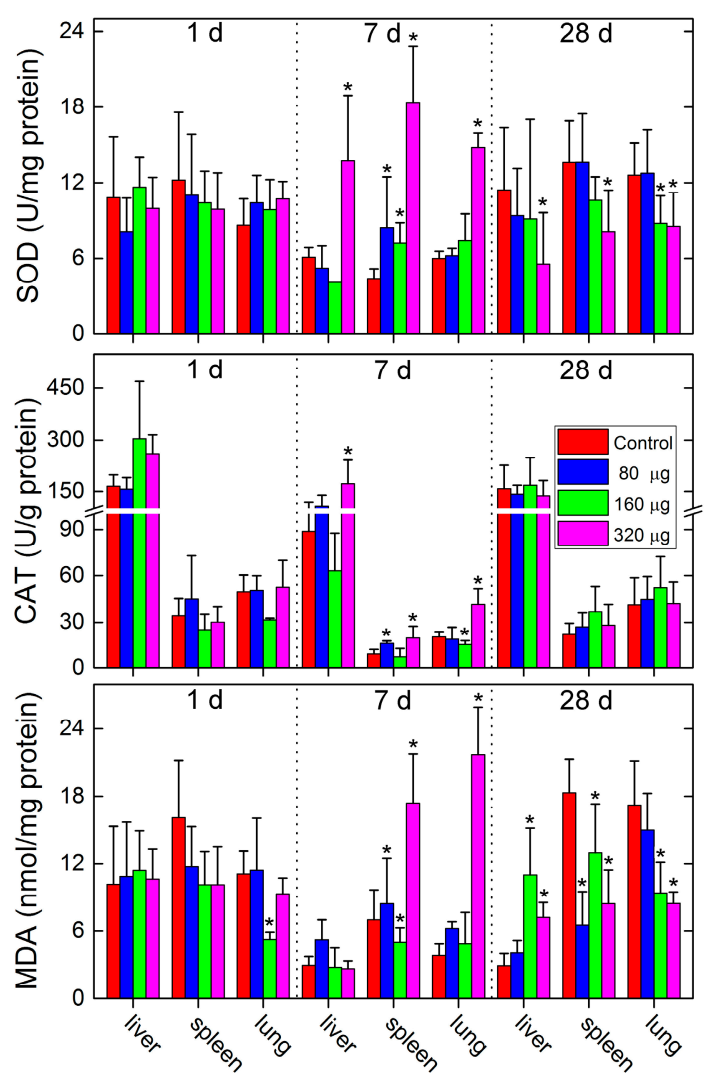

Figure 6. Oxidative stress levels of the mice exposed to CNSI post intravenous exposure. Data represent means $\pm \mathrm{SD}(n=5) .{ }^{*} p<0.05$ compared with the control group.

\section{Materials and Methods}

\subsection{Materials}

Commercial CNSI (50 mg/mL) was provided by Chongqing Lummy Pharmaceutical Co., Ltd, Chongqing, China. All kits for serum biochemistry were obtained from MSKBio Science and Technology Co., Wuhan, China. All reagents for the TUNEL method were purchased from Beijing Dingguo Changsheng Biotechnology Co., Beijing, China. All kits for oxidative stress assays were obtained from the Nanjing Jiancheng Bioengineering Institute, Nanjing, China. Other chemicals were of analytical grade and used without purification.

\subsection{Characterization of CNSI}

CNSI was carefully characterized by TEM (Autoflex, Bruker, Bonn, Germany), DLS (Zetasizer Nano ZS90, Malvern Instruments, Malvern, UK), and Raman spectroscopy (Renishaw inVia plus, Renishaw, Wotton-under-Edge, UK) before use. Carbon nanoparticles without adding suspending reagents were analyzed by XPS (Axis Ultra, Kratos, Manchester, UK) and IR (Tensor27, Bruker, Germany). The C1s spectrum was analyzed by CasaXPS software (version 2.3.15, Casa Software Ltd., Knutsford, UK) following the automatic fitting protocol.

\subsection{Animal Exposure}

The animal experiments were checked and approved by the Animal Center of Southwest Minzu University. The experiments were performed strictly in accordance with the Animal Care and Use Program Guidelines of Sichuan Province, China. Institute of Cancer Research (ICR) mice ( $25 \mathrm{~g})$ were purchased from Dashuo Experimental Animal Co., Chengdu, China, and raised in plastic cages 
(six mice/cage) on a 12-h light/dark cycle with ad libitum access to food and water. After the acclimation, the mice were randomly divided into groups of six mice for each CNSI exposure.

CNSI and saline were filtered with $0.22-\mu \mathrm{m}$ filters for sterilization before use. Mice injected intravenously with $0.2 \mathrm{~mL}$ of saline solution in one injection were taken as the control group. The human dosage $(0.71 \mathrm{mg} / \mathrm{kg}$ body weight) was multiplied with a factor for mice (9.1), resulting in the middle dosage of $6.5 \mathrm{mg} / \mathrm{kg}$ body weight for mice. Mice injected with $80 \mu \mathrm{g}$ carbon nanoparticles per mouse $(3.2 \mathrm{mg} / \mathrm{kg}$ body weight) in one injection were set as the $80 \mu \mathrm{g}$ group. Mice injected with $160 \mu \mathrm{g}$ carbon nanoparticles per mouse $(6.5 \mathrm{mg} / \mathrm{kg}$ body weight) in one injection were set as the $160 \mu \mathrm{g}$ group. Mice injected with $320 \mu \mathrm{g}$ carbon nanoparticles per mouse $(13 \mathrm{mg} / \mathrm{kg}$ body weight) in one injection were set as the $320 \mu \mathrm{g}$ group. For CNSI injection, the CNSI solution was diluted with saline to ensure the injection volume of $0.2 \mathrm{~mL}$. After the injection, the behaviors of mice were recorded daily and the bodyweights were measured with the interval of 3 days. Before the sacrifice at 1,7, and 28 days, the mice were fasted for $12 \mathrm{~h}$. After collecting blood, the mice were sacrificed by cervical dislocation.

\subsection{Biodistribution of CNSI}

First, we checked the histopathological samples after HE staining under an optical microscope. The heart, liver, spleen, lungs, kidneys and axillary lymph nodes of the $320 \mu \mathrm{g}$ group at 1 day were fixed by $4 \%$ paraformaldehyde solution. The fixed samples were embedded in paraffin, thin-sectioned, and mounted on glass microscope slides using the standard histopathological techniques. The mounted sections were stained with HE for optical microscopy. Black or brown spots were carefully checked and recorded.

For Raman analyses, the tissue samples were grounded with deionized water $(0.1 \mathrm{~g}$ tissue in $0.1 \mathrm{~mL}$ water) with a homogenizer. The homogenate samples were placed on glass slides and directly analyzed by the Raman spectrometer. The parameters of Renishaw micro-Raman spectroscopy system were set as: laser excitation wavelength $785 \mathrm{~nm}, 50 \mathrm{~mW}$ power, $50 \times$ objective, laser spot size $50 \times 2 \mu \mathrm{m}^{2}$, $1 \mathrm{~s}$ collection time, data accumulation of 100 times. The baseline and smoothing were automatically performed with the software.

\subsection{Toxicity Evaluations}

For hematological analysis, the blood samples were combined with $0.1 \mathrm{~mL} 15 \mathrm{~g} / \mathrm{L}$ ethylenediaminetetraacetic acid dipotassium salt $\left(\right.$ EDTA- $\left.\mathrm{K}_{2}\right)$ for anticoagulation immediately after blood collection. The hematological measurements were performed on an automatic hematology analyzer (BC-5800, Mindray Co., Shenzhen, China) following the standard protocols. For serum biochemistry analysis, the blood samples were kept at room temperature for $1 \mathrm{~h}$ and then centrifuged at $3338 \mathrm{~g}$ for $10 \mathrm{~min}$ to collect the supernatant. The biochemical assays were performed on a clinical automatic chemistry analyzer (Chemray360, Rayto Co., Shenzhen China) following the standard protocols.

For histopathological observations, the samples of control and CNSI-exposed groups were fixed, sectioned, and stained by HE as described above. The samples were checked under an optical microscope. For apoptosis evaluations, the TUNEL method was adopted. The slides were prepared strictly following the manufacturer's instructions and observed under a light microscope. The detailed protocol is presented at the official website of the manufacturer (online resource) [36].

For oxidative stress assays, each sample was minced and homogenized in $4{ }^{\circ} \mathrm{C}$ saline three times (10 s/time, intermittent for $30 \mathrm{~s})$ to yield $10 \%(w / v)$ homogenate. The homogenates were centrifuged at $2225 \times g$ for $10 \mathrm{~min}$ to obtain the supernatants. Protein concentrations in the supernatants were determined according to the method of Bradford, using bovine serum albumin as the standard. The SOD, CAT, and MDA levels were analyzed following the manufacturer's instructions using an UV-vis spectrophotometer (UV-1800, Mapada, Shanghai, China). The detailed protocols can be found at the official website of the manufacturer (online resource) [37]. 


\subsection{Statistical Analysis}

All data were expressed as the means of six individual samples with standard deviation (means $\pm \mathrm{SD}$ ). Significance was calculated by using the Student's $t$-test method, where $p<0.05$ was taken as statistically significant.

\section{Conclusions}

In summary, the accumulation and toxicity of CNSI were preliminarily evaluated after intravenous injection in mice, where CNSI was trapped in RES organs and no apparent toxicity was observed. CNSI accumulated majorly in the liver and spleen after intravenous injection, while only very small amounts were detected in the lungs. The mice behaved normally and their body weight increases were not disturbed upon exposure to CNSI. The nearly unchanged hematological and serum biochemical parameters indicated the low toxicity of CNSI in vivo, which was further confirmed by the histopathological observations and apoptosis analyses. The only hazard of CNSI was the induction of oxidative stress in mice. Overall, the low toxicity of CNSI after intravenous exposure ensures safe theranostics applications in the future and also confirms the biosafety of CNSI that entered the blood circulation during the intratumoral injection. It is hoped that our results will benefit the ongoing exploration of the clinical applications and biosafety evaluations of carbon-based nanomaterials.

Acknowledgments: This work was supported by the major drug discovery science and technology major projects of the 12th five-year national plan and research fund for major drug research of Nation Science and Technology (863 Projects, 2012ZX09102001-4 and 2012ZX09102101-015), National Program for Support of Top-notch Young Professionals, and the Fundamental Research Funds for the Central Universities, Southwest Minzu University (No. 2016NZDFH01).

Author Contributions: Sheng-Tao Yang and Xiao-Hai Tang conceived and designed the experiments; Ping Xie, Tiantian He, and Shengnan Yang performed the experiments; Ping Xie, Tiantian He, Shengnan Yang, and Sheng-Tao Yang analyzed the data; Sheng-Tao Yang and Xiao-Hai Tang wrote the paper.

Conflicts of Interest: Tiantian He and Xiao-Hai Tang are employees of Chongqing Lummy Pharmaceutical Co., Ltd. The authors report no other conflicts of interest in this work. The founding sponsors had no role in the design of the study; in the collection, analyses, or interpretation of data; in the writing of the manuscript, and in the decision to publish the results.

\section{Abbreviations}

$\begin{array}{ll}\text { CNSI } & \text { Carbon nanoparticles suspension injection } \\ \text { RES } & \text { Reticuloendothelial system } \\ \text { CNTs } & \text { Carbon nanotubes } \\ \text { TDLN } & \text { Tumor drainage lymph node } \\ \text { GO } & \text { Graphene oxide } \\ \text { TUNEL } & \text { Terminal deoxynucleotidyl transferase-mediated dUTP nick-end labeling } \\ \text { PVP } & \text { Polyvinyl pyrrolidone } \\ \text { TEM } & \text { Transmission electron microscopy } \\ \text { DLS } & \text { Dynamic light scattering } \\ \text { XPS } & \text { X-ray photoelectron spectroscopy } \\ \text { FWHM } & \text { Full width at half maximum } \\ \text { IR } & \text { Infrared spectroscopy } \\ \text { HE } & \text { Hematoxylin-eosin } \\ \text { HB } & \text { Hemoglobin } \\ \text { MCV } & \text { Mean corpuscular volume } \\ \text { MCHC } & \text { Mean corpuscular hemoglobin } \\ \text { MPV } & \text { Mean platelet volume } \\ \text { PLT } & \text { Platelet } \\ \text { WBC } & \text { White blood cell count }\end{array}$




$\begin{array}{ll}\text { RBC } & \text { Red blood cell count } \\ \text { MCH } & \text { Mean corpuscular hemoglobin } \\ \text { RDW } & \text { Red cell distribution width } \\ \text { PDW } & \text { Platelet distribution width } \\ \text { TBIL } & \text { Total bilirubin } \\ \text { LDH } & \text { Lactate dehydrogenase } \\ \text { ALT } & \text { Alanine aminotransferase } \\ \text { AST } & \text { Aspartate aminotransferase } \\ \text { ALP } & \text { Alkaline phosphatase } \\ \text { Ur } & \text { Urea } \\ \text { Cr } & \text { Creatinine } \\ \text { SOD } & \text { Superoxide dismutase } \\ \text { CAT } & \text { Catalase } \\ \text { MDA } & \text { Malondialdehyde } \\ \text { ICR } & \text { Institute of Cancer Research }\end{array}$

\section{References and Notes}

1. LeCroy, G.E.; Yang, S.-T.; Yang, F.; Yang, F.; Liu, Y.; Fernando, K.A.S.; Bunker, C.E.; Hu, Y.; Luo, P.G.; Sun, Y.-P. Functionalized carbon nanoparticles: Syntheses and applications in optical bioimaging and energy conversion. Coord. Chem. Rev. 2016, 320-321, 66-81. [CrossRef]

2. De Volder, M.F.L.; Tawfick, S.H.; Baughman, R.H.; Hart, A.J. Carbon nanotubes: Present and future commercial applications. Science 2013, 339, 535-539. [CrossRef] [PubMed]

3. Hong, G.; Diao, S.; Antaris, A.L.; Dai, H. Carbon nanomaterials for biological imaging and nanomedicinal therapy. Chem. Rev. 2015, 115, 10816-10906. [CrossRef] [PubMed]

4. Luo, P.G.; Yang, F.; Yang, S.-T.; Sonkar, S.K.; Yang, L.; Broglie, J.J.; Liu, Y.; Sun, Y.-P. Carbon-based quantum dots for fluorescence imaging of cells and tissues. RSC Adv. 2014, 4, 10791-10807. [CrossRef]

5. Son, K.H.; Hong, J.H.; Lee, J.W. Carbon nanotubes as cancer therapeutic carriers and mediators. Int. J. Nanomed. 2016, 11, 5163-5185. [CrossRef] [PubMed]

6. Zhang, B.; Wang, Y.; Liu, J.; Zhai, J. Recent developments of phototherapy based on graphene family nanomaterials. Curr. Med. Chem. 2017, 24, 268-291. [CrossRef] [PubMed]

7. Wang, P.; Liu, J.; Gao, H.; Hu, Y.; Hou, X.; LeCroy, G.E.; Bunker, C.E.; Liu, Y.; Sun, Y.-P. Host-guest carbon dots as high-performance fluorescence probes. J. Mater. Chem. C 2017, 5, 6328-6335. [CrossRef]

8. Li, Y.; Zheng, X.; Zhang, X.; Liu, S.; Pei, Q.; Zheng, M.; Xie, Z. Porphyrin-based carbon dots for photodynamic therapy of hepatoma. Adv. Healthc. Mater. 2017, 6, 1600924. [CrossRef] [PubMed]

9. Reina, G.; González-Domínguez, J.M.; Criado, A.; Vázquez, E.; Bianco, A.; Prato, M. Promises, facts and challenges for graphene in biomedical applications. Chem. Soc. Rev. 2017, 46, 4400-4416. [CrossRef] [PubMed]

10. Chongqing Lummy Pharmaceutical Co., Ltd., Chongqing, China. Internal data of carbon nanoparticles suspension injection, 2017.

11. Li, Z.; Ao, S.; Bu, Z.; Wu, A.; Wu, X.; Shan, F.; Ji, X.; Zhang, Y.; Xing, Z.; Ji, J. Clinical study of harvesting lymph nodes with carbon nanoparticles in advanced gastric cancer: A prospective randomized trial. World J. Surg. Oncol. 2016, 14, 88. [CrossRef] [PubMed]

12. Wu, X.; Lin, Q.; Chen, G.; Lu, J.; Zeng, Y.; Chen, X.; Yan, J. Sentinel lymph node detection using carbon nanoparticles in patients with early breast cancer. PLoS ONE 2015, 10, e0135714. [CrossRef] [PubMed]

13. Zhu, Y.; Chen, X.; Zhang, H.; Chen, L.; Zhou, S.; Wu, K.; Wang, Z.; Kong, L.; Zhuang, H. Carbon nanoparticle-guided central lymph node dissection in clinically node-negative patients with papillary thyroid carcinoma. Head Neck 2016, 38, 840-845. [CrossRef] [PubMed]

14. Gu, J.; Wang, J.; Nie, X.; Wang, W.; Shang, J. Potential role for carbon nanoparticles identification and preservation in situ of parathyroid glands during total thyroidectomy and central compartment node dissection. Int. J. Clin. Exp. Med. 2015, 8, 9640-9648. [PubMed] 
15. Xie, P.; Tang, X.; Li, L.; Qian, Z.; Ran, M.; Zhang, X.; Xin, Q.; Luo, H. Drug-loaded carbon nanoparticle suspension injection, Drug selection, releasing behavior, in vitro cytotoxicity and in vivo lymph node targeting. J. Nanosci. Nanotechnol. 2016, 16, 6910-6918. [CrossRef]

16. Yang, Q.; Wang, X.; Chen, J.; Tian, C.; Li, T.; Chen, Y.; Lv, C. A clinical study on regional lymphatic chemotherapy using an activated carbon nanoparticle-epirubicin in patients with breast cancer. Tumor Biol. 2012, 33, 2341-2348. [CrossRef] [PubMed]

17. Xie, P.; Xin, Q.; Yang, S.-T.; He, T.; Huang, Y.; Zeng, G.; Ran, M.; Tang, X.H. Skeleton labeled ${ }^{13}$ C-carbon nanoparticles for the imaging and quantification in tumor drainage lymph nodes. Int. J. Nanomed. 2017, 12, 4891-4899. [CrossRef] [PubMed]

18. Liu, Y.; Zhao, Y.; Sun, B.; Chen, C. Understanding the toxicity of carbon nanotubes. Acc. Chem. Res. 2013, 46, 702-713. [CrossRef] [PubMed]

19. Chang, X.; Yang, S.-T.; Xing, G. Molecular toxicity of nanomaterials. J. Biomed. Nanotechnol. 2014, 10, 2828-2851. [CrossRef] [PubMed]

20. Yang, S.-T.; Luo, J.; Zhou, Q.; Wang, H. Pharmacokinetics, metabolism and toxicity of carbon nanotubes for biomedical purposes. Theranostics 2012, 2, 271-282. [CrossRef] [PubMed]

21. Yang, S.-T.; Wang, X.; Jia, G.; Gu, Y.; Wang, T.; Nie, H.; Ge, C.; Wang, H.; Liu, Y. Long-term accumulation and low toxicity of single-walled carbon nanotubes in intravenously exposed mice. Toxicol. Lett. 2008, 181, 182-189. [CrossRef] [PubMed]

22. Yang, S.-T.; Wang, X.; Wang, H.; Lu, F.; Luo, P.G.; Cao, L.; Meziani, M.J.; Liu, J.-H.; Liu, Y.; Chen, M.; et al. Carbon dots as nontoxic and high performance fluorescence imaging agents. J. Phys. Chem. C 2009, 113, 18110-18114. [CrossRef] [PubMed]

23. Liu, J.; Wang, T.; Wang, H.; Gu, Y.; Xu, Y.; Tang, H.; Jia, G.; Liu, Y. Biocompatibility of graphene oxide intravenously administrated in mice-effects of dose, size and exposure protocols. Toxicol. Res. 2015, 4, 83-91. [CrossRef]

24. Kanakia, S.; Toussaint, J.D.; Chowdhury, S.M.; Tembulkar, T.; Lee, S.; Jiang, Y.; Lin, R.Z.; Shroyer, K.R.; Moore, S.; Sitharaman, B. Dose ranging, expanded acute toxicity and safety pharmacology studies for intravenously administered functionalized graphene nanoparticle formulations. Biomaterials 2014, 35, 7022-7031. [CrossRef] [PubMed]

25. Zhang, D.; Deng, X.; Ji, Z.; Dong, L.; Wu, M.; Gu, T.; Liu, Y. Long-term hepatotoxicity of polyethylene-glycol functionalized multi-walled carbon nanotubes in mice. Nanotechnology 2010, 21, 175101. [CrossRef] [PubMed]

26. Wang, H.; Yang, S.-T.; Cao, A.; Liu, Y. Quantification of carbon nanomaterials in vivo. Acc. Chem. Res. 2013, 46, 750-760. [CrossRef] [PubMed]

27. Choi, H.S.; Liu, W.; Misra, P.; Tanaka, E.; Zimmer, J.P.; Ipe, B.I.; Bawendi, M.G.; Frangioni, J.V. Renal clearance of quantum dots. Nat. Biotechnol. 2007, 25, 1165-1170. [CrossRef] [PubMed]

28. Owens, D.E.; Peppas, N.A. Opsonization, biodistribution, and pharmacokinetics of polymeric nanoparticles. Int. J. Pharm. 2006, 307, 93-102. [CrossRef] [PubMed]

29. Chongqing Lummy Pharmaceutical Co., Ltd., Chongqing, China. Histopathological observations of mice injected with carbon nanoparticles suspension injection at day 1 and 7, 2017.

30. Snyder-Talkington, B.N.; Dong, C.; Porter, D.W.; Ducatman, B.; Wolfarth, M.G.; Andrew, M.; Battelli, L.; Raese, R.; Castranova, V.; Guo, N.L.; et al. Multiwalled carbon nanotube-induced pulmonary inflammatory and fibrotic responses and genomic changes following aspiration exposure in mice: A 1-year post-exposure study. J. Toxicol. Environ. Health A 2016, 79, 352-366. [CrossRef] [PubMed]

31. Bengtson, S.; Knudsen, K.B.; Kyjovska, Z.O.; Berthing, T.; Skaug, V.; Levin, M.; Koponen, I.K.; Shivayogimath, A.; Booth, T.J.; Alonso, B.; et al. Differences in inflammation and acute phase response but similar genotoxicity in mice following pulmonary exposure to graphene oxide and reduced graphene oxide. PLoS ONE 2016, 12, e0178355. [CrossRef] [PubMed]

32. Zhang, J.Q.; Sun, Q.; Bo, J.; Huang, R.; Zhang, M.; Xia, Z.; Ju, L.; Xiang, G. Single-walled carbon nanohorn $(\mathrm{SWNH})$ aggregates inhibited proliferation of human liver cell lines and promoted apoptosis, especially for hepatoma cell lines. Int. J. Nanomed. 2014, 9, 759-773. [CrossRef] [PubMed]

33. Chongqing Lummy Pharmaceutical Co., Ltd., Chongqing, China. TUNEL assays of mice injected with carbon nanoparticles suspension injection at day 1 and 7, 2017.

34. Shvedova, A.A.; Pietroiusti, A.; Fadeel, B.; Kagan, V.E. Mechanisms of carbon nanotube-induced toxicity: Focus on oxidative stress. Toxicol. Appl. Pharmcol. 2012, 261, 121-133. [CrossRef] [PubMed] 
35. Nurunnabi, M.; Khatun, Z.; Huh, K.M.; Park, S.Y.; Lee, D.Y.; Cho, K.J.; Lee, Y. In vivo biodistribution and toxicology of carboxylated graphene quantum dots. ACS Nano 2013, 7, 6858-6867. [CrossRef] [PubMed]

36. Available online: http://www.dingguo.com/Asset/emspics/UP_2012051107034911.pdf (accessed on 3 March 2017).

37. Available online: http:/ / elder.njjcbio.com/index_en.php (accessed on 3 March 2017).

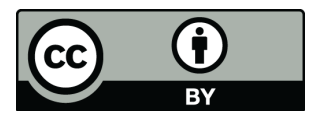

(C) 2017 by the authors. Licensee MDPI, Basel, Switzerland. This article is an open access article distributed under the terms and conditions of the Creative Commons Attribution (CC BY) license (http:/ / creativecommons.org/licenses/by/4.0/). 